Gut, 1975, 16, 985-987

\title{
Serum lysozyme in inflammatory bowel disease
}

\author{
M. W. DRONFIELD AND M. J. S. LANGMAN \\ From the Department of Therapeutics, City Hospital, Nottingham
}

SUMMARY The mean concentrations of serum lysozyme were markedly higher in patients with Crohn's disease and ulcerative colitis than in normal controls, and mean levels tended to be slightly higher in those with Crohn's disease than in those with colitis. The significance of these differences is unclear but the overlap between values in normal individuals and those with inflammatory bowel disease prevents the measurement having any discriminant value.

Raised serum levels of lysozyme (muramidase) have recently been detected in patients with Crohn's disease (Falchuk et al., 1975), there being no overlap of levels between Crohn's disease patients and patients with ulcerative colitis or normal controls, suggesting that such determinations may be useful in differentiating between inflammatory bowel diseases. We have therefore sought to confirm or deny this clear differentiation.

\section{Methods}

\section{PATIENTS}

Twenty-seven patients with Crohn's disease were studied (five men and 22 women, mean age 38 years). Nine had small intestinal disease, 10 ileocolonic disease, and five colonic disease only, the remaining three having had successful intestinal resections. Twenty-five patients (10 men and 15 women, mean age 42 years) had ulcerative colitis as judged by conventional criteria, five had total colonic disease, and 19 left-sided disease only, the remaining patient having had a total colectomy. Patients with disease that could not be clearly classified have been excluded from this report.

Controls consisted of 26 healthy volunteers (19 men and seven women, mean age 24 years) and 11 patients with chronic renal failure due to a variety of causes, all with blood urea levels above $100 \mathrm{mg} \%$.

LYSOZYME DETERMINATION

A suspension of killed Micrococcus lysodeikticus (Sigma) was made up in $66.7 \mathrm{mM}$ phosphate buffer at pH 6.24, so that an optical density of around 0.7 was achieved, as measured on a Gilford model 240 spectrophotometer attached to a Kontron chart

Received for publication 15 October 1975. recorder, a concentration of approximately $13 \mathrm{mg} /$ $100 \mathrm{ml}$ of micrococcus being required to achieve this optical density. To $2.5 \mathrm{ml}$ of this suspension was added $0.1 \mathrm{ml}$ serum, and the fall in optical density over two minutes at $37^{\circ} \mathrm{C}$ was recorded. The lytic activity thus obtained was compared with that obtained by known concentrations of egg white lysozyme (Sigma) in concentrations ranging from 5 to $40 \mu \mathrm{g} / \mathrm{ml}$, and the equivalent lysozyme level in each sample of serum was thus deduced. Most measurements were done on the day of venesection, and all were done within $\mathbf{4 8}$ hours. Sera were stored at $-20^{\circ} \mathrm{C}$ if any delay occurred between venesection and measurement.

Results obtained by the above technique were compared in 26 individuals including normal controls, uraemic controls, and ulcerative colitis and Crohn's disease patients, with results obtained by the lysoplate method (Osserman and Lawlor, 1966). We used $1 \%$ agarose (BDH Chemicals) containing Micrococcus lysodeikticus at a concentration of 70 $\mathrm{mg} / 100 \mathrm{ml}$ made up in $66.7 \mathrm{mM}$ phosphate buffer at $\mathrm{pH} \mathrm{6.24}$. This was placed in a $10 \mathrm{~cm} \times 10 \mathrm{~cm}$ square plate (Sterilin products) to a depth of $3 \mathrm{~mm}$, and wells $2 \mathrm{~mm}$ in diameter were cut out in the agar; $5 \mu \mathrm{l}$ of serum or control egg white lysozyme were put into each well and the plate was then left to incubate overnight at room temperature. Clear circles of lysis in the opalescent agar developed around each well and were measured by projection onto paper with a drawing tube attachment on a Leitz microscope. The serum values were then obtained by comparison with the known control egg white lysozyme levels.

\section{Results}

The lysozyme levels obtained spectrohhotometrically are shown in the Figure. Mean concentrations were 


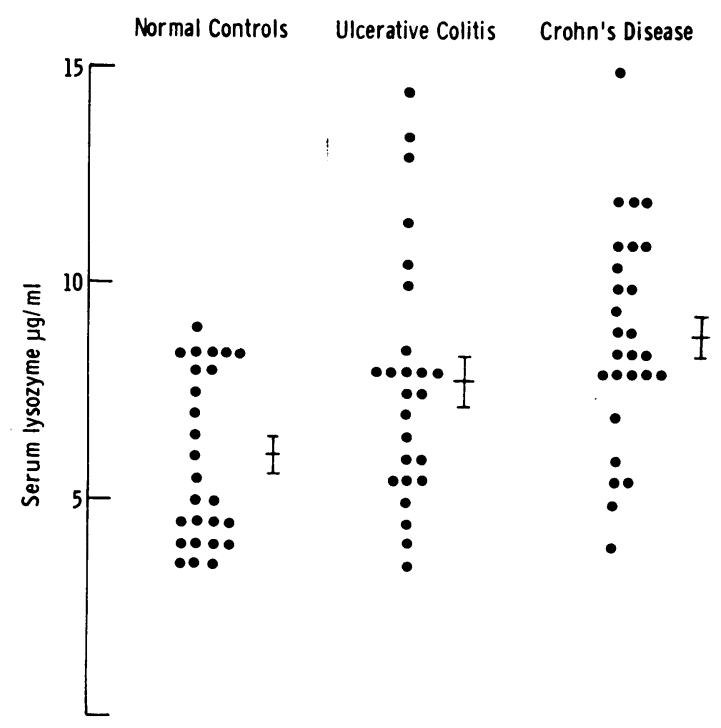

Figure Serum lysozyme concentration in patients and controls. Mean levels (horizontal bar) and standard error of the mean are shown.

significantly higher in patients with Crohn's disease and ulcerative colitis than in the normal controls ( $P<0.001$ and $P<0.02$ respectively). Higher levels than those found in any of the controls were obtained in 11 of the 27 patients with Crohn's disease, and six of the 25 with ulcerative colitis, the remaining values all falling within the normal range. Though mean lysozyme concentrations were slightly higher in the Crohn's disease patients than in the ulcerative colitis group, the difference could well have been due to chance $(P>0 \cdot 1)$. As expected, since catabolism of lysozyme occurs mainly within the kidneys (Hansen et al., 1972), considerably raised values were found in the 11 patients with renal failure (mean $23.5 \mu \mathrm{g} / \mathrm{ml}$, range 11 to $49 \mu \mathrm{g} / \mathrm{ml}$ ).

Since lysozyme in the serum originates, in normal circumstances, from circulating neutrophils and monocytes, counts of these cells were compared in the patients with ulcerative colitis and Crohn's disease. Neutrophil and monocyte counts were on average slightly higher in the Crohn's disease group than in those with colitis (means 5945 and 5037 cells $/ \mathrm{mm}^{3}$ and 525 and 516 cells $/ \mathrm{mm}^{3}$ respectively), but there was no detectable correlation between neutrophil or monocyte counts and serum lysozyme levels, except that both monocyte counts and serum lysozyme levels were very high (1044 and 1335 cells/ $\mathrm{mm}^{3}$ and 13.0 and $13.5 \mu \mathrm{g} / \mathrm{ml}$ respectively) in two patients with ulcerative colitis.

No correlation was detectable in either the ulcerative colitis or Crohn's disease patients between lysozyme levels and the patient's sex and age or the activity, duration, or extent of their disease.

The results obtained by the lysoplate method correlated well with those obtained spectrophotometrically $(r=0.89: \mathrm{P}<0.001)$. The lysoplate concentrations expressed as $\mu \mathrm{g} / \mathrm{ml}$ derived from comparison with egg white lysozyme were approximately three times as high as levels obtained on the spectrophotometer, in keeping with the experience of Osserman and Lawler (1966).

\section{Discussion}

Though serum lysozyme levels tended to be higher in Crohn's disease than in ulcerative colitis, and in both of these than in normal controls, no clear discriminant pattern emerged. Our findings therefore differ from those of Falchuk et al. (1975) who suggested that serum lysozyme levels would be useful in distinguishing Crohn's disease from ulcerative colitis. They used the lysoplate technique with human lysozyme as the control, whereas we have mainly used a spectrophotometric method with egg white lysozyme as the control. However, we have obtained a good correlation between the spectrophotometric and lysoplate techniques, and there seems no reason for believing that the use of egg white lysozyme rather than human lysozyme should obscure real differences in lysozyme concentrations between patient groups. Furthermore, we have, as expected, been able to find high levels in patients with renal failure who thus constitute a positive control.

High lysozyme levels have been detected in patients with granulomatous diseases such as tuberculosis and sarcoidosis in the past (Pascual et al., 1973; Perillie et al., 1973), but the distribution of values has also overlapped that of the controls.

It seems likely that the raised enzyme levels detectable in some of our patients were due to lysozyme derived from tissue macrophages and epithelioid cells. No correlation was detectable between serum lysozyme and blood monocyte or neutrophil counts, the usual source of serum lysozyme (Fink and Finch, 1968). In addition, faecal lysozyme levels have been found to be raised in both ulcerative colitis (Meyer et al., 1948) and Crohn's disease (Gray et al., 1950).

More recently high concentrations of lysozyme have been found in the epithelioid histiocytes and giant cells of the granulomata of patients with Crohn's disease, sarcoidosis and tuberculosis (Mason and Taylor, 1975). Ulcerative colitis tissue was not examined. The significance of the raised serum lysozyme levels found in some of our patients is 
unclear. Though we could detect no correlation with disease type, extent and duration, it remains possible that the clinical course of patients with high and low levels will ultimately differ.

We are grateful to Mr. A. Brammall for his technical assistance. M.W.D. is in receipt of a grant from Fisons Limited, England, which is gratefully acknowledged.

\section{References}

Falchuk, K. R., Perrotto, J. L., and Isselbacher, K. J. (1975). Serum lysozyme in Crohn's disease and ulcerative colitis. New England Journal of Medicine, 292, 395-397.

Fink, M. E., and Finch, S. C. (1968). Serum muramidase and granulocyte turnover. Proceedings of the Society for Experimental Biology, 127, 365-367.

Gray, S. J., Reifenstein, R. W., Connolly, E. P., Spiro,
H. M., and Young, J. C. G. (1950). Studies on lysozyme in chronic ulcerative colitis. Gastroenterology, 16, 687-696. Hansen, N. E., Karle, H., Andersen, V., and Ølgard, K. (1972). Lysozyme turnover in man. Journal of Clinical Investigation, 51, 1146-1155.

Mason, D. Y., and Taylor, C. R. (1975). The distribution of muramidase (lysozyme) in human tissues. Journal of Clinical Pathology, 28, 124-132.

Meyer, K., Gellhorn, A., Prudden, J. F., Lehman, W. L., and Steinberg, A. (1948). Lysozyme activity in ulcerative alimentary disease. American Journal of Medicine, 5, 496502.

Osserman, E. F., and Lawlor, D. P. (1966). Serum and urinary lysozyme (muramidase) in monocytic and monomyelocytic leukemia. Journal of Experimental Medicine, 124, 921-952.

Pascual, R. S., Gee, J. B. L., and Finch, S. C. (1973). Usefulness of serum lysozyme measurement in diagnosis and evaluation of sarcoidosis. New England Journal of Medicine, 289, 1074-1076.

Perillie, P. E., Khan, K., and Finch, S. C. (1973). Serum lysozyme in pulmonary tuberculosis. American Journal of Medical Science, 265, 297-302. 